\title{
AVALIAÇÃo PSICOLÓgICA: PASSADO, PRESENTE E FUTURO
}

\author{
Luciana França Cescon \\ Graduada em Psicologia pela Universidade Paulista, pós-graduada em Saúde Pública pela Atualize, \\ mestranda em Ciências da Saúde pela Unifesp.
}

\section{Resumo}

O presente artigo tem como objetivo contribuir para a discussão da questão da avaliação psicológica que se faz hoje e a que se deseja realizar no século XXI, tendo como ponto principal as contribuições que a Psicologia Sócio-Histórica tem trazido, bem como os desafios a serem superados para uma prática cada vez mais transformadora dentro do contexto social. Acreditamos que a avaliação psicológica utilizada dentro de um processo que considere em sua análise os fatores sociais e históricos que afetam a vida das pessoas possa contribuir para o fortalecimento das potencialidades e autonomia das mesmas. Utilizando os dados obtidos na avaliação psicológica de forma construtiva, como ferramenta de informação e transformação, os sujeitos atendidos podem ter acesso a instrumentos necessários para o enfrentamento de suas dificuldades, passando a avaliação psicológica a contribuir de forma significativa para a consolidação dos direitos humanos e o respeito à identidade dos indivíduos.

Palavras-chave: avaliação psicológica; psicologia sócio-histórica.

\section{PSYCHOLOGICAL ASSESSMENT: PAST, PRESENT AND FUTURE}

\begin{abstract}
This paper aims to contribute to the discussion of the issue of psychological assessment that is done today and to be carried out in the 21st century, having as main point the contributions that the Socio-Historical Psychology has brought, as well as challenges to overcome for a practice increasingly transforming within the social context. We believe that the psychological evaluation used within a process to consider in its analysis the social and historical factors that affect people's lives can contribute to strengthening the potential and empower them. Using the data obtained in psychological assessment constructively, as an information tool and transformation, the subjects may have access to the necessary tools to cope with their difficulties, from the psychological evaluation to contribute significantly to the consolidation of human rights and with the identity of individuals.
\end{abstract}

Keywords: psychological assessment; socio-historical psychology. 


\title{
EVALUACIÓN PSICOLÓgICA: PASADO, PRESENTE Y FUTURO
}

\begin{abstract}
Resumen
Este artículo tiene como objetivo contribuir a la discusión de la cuestión de la evaluación psicológica que se hace hoy y que se llevará a cabo en el siglo XXI, teniendo como punto principal de las aportaciones que la psicología socio-histórica ha traído, así como los retos que hay que superar una práctica cada vez más de transformación en el contexto social. Creemos que la evaluación psicológica utilizada dentro de un proceso para considerar en su análisis de los factores históricos y sociales que afectan la vida de las personas puede contribuir a reforzar el potencial y la autonomía de ellos. Con los datos obtenidos en la evaluación psicológica de manera constructiva, como herramienta de información y de transformación, los sujetos atendidos pueden tener acceso a las herramientas necesarias para hacer frente a sus dificultades, a partir de la evaluación psicológica para contribuir significativamente a la consolidación de los derechos humanos y con la identidad de los individuos.

Palabras clave: evaluación psicológica; psicología socio-histórica.
\end{abstract}

\section{INTRODUÇÃO}

Falar sobre avaliação psicológica é refletir sobre a importância de uma das principais funções do profissional de Psicologia. Historicamente, a imagem que se tem do psicólogo é a do profissional que se utiliza de testes, que avalia "se uma pessoa é normal ou não", se está apta a executar determinada função, já que a testagem foi uma das atividades mais comuns no século XX no campo da Psicologia (Cunha, 2000), como discutiremos mais adiante. Porém, a prática psicológica tem se expandido cada vez mais, e neste movimento, a avaliação psicológica passou a ser bem mais abrangente, considerando diversos fatores em seu processo de estudo e análise dos sujeitos.

De acordo com a Resolução do Conselho Federal de Psicologia no. 007 (2003), pode-se definir a avaliação psicológica como sendo o processo técnicocientífico de coleta de dados, estudos e interpretação de informações a respeito dos fenômenos psicológicos da relação do indivíduo com a sociedade. Utiliza-se, para isto, de estratégias psicológicas - métodos, técnicas e instrumentos. Os resultados das avaliações devem considerar e analisar os condicionantes históricos e sociais e seus efeitos no psiquismo, com a finalidade de servirem como instrumentos para atuar não somente sobre o indivíduo, mas em todo o contexto social e histórico no qual ele se insere.

Na prática, agregar todas estas informações em uma análise que seja capaz de utilizá-las de forma integradora e dialética ainda é um desafio. O debate e a reflexão sobre o papel do psicólogo nesta questão são extremamente importantes, uma vez que é necessário manter uma visão crítica que vai além da 
concepção de investigar o que é patológico ou não, e passa a ter como enfoque a compreensão acerca da subjetividade de determinado indivíduo, visando seu benefício. O psicólogo deve buscar, da melhor maneira possível, apreender a relação entre saúde mental e subjetividade, considerando sua dimensão social. Porém, estamos tão acostumados a pensar em utilizar a avaliação psicológica com o objetivo de chegar a um diagnóstico, que às vezes fica difícil conseguirmos separar o que é individual e o que é social.

"Aceitar diferenças individuais, mantendo relações de igualdade, ou melhor, de não dominação, em uma sociedade onde as diferenças são valorizadas em termos de competição, torna-se algo extremamente difícil". (Lane, 1987, p. 72).

Este trabalho pretende trazer uma análise sobre a avaliação psicológica desde sua origem e os desafios que devem ser superados para que ela tenha cada vez mais um caráter de transformação social.

\section{A avaliação psicológica que se faz}

Para contextualizarmos esta discussão, falaremos brevemente sobre o surgimento da Psicologia como ciência. Sua origem data de 1875, com Wundt, para quem o objeto de estudo da Psicologia seria a experiência imediata dos sujeitos. Influenciada pela característica das ciências do século XIX, o caráter inicial da ciência psicológica é positivista e racionalista. Wundt sugere então a criação de duas vertentes do campo psicológico: a Psicologia Experimental, que reconhece a causalidade psíquica (mas não a investiga em profundidade), e a Psicologia Social ou "dos povos", que procura estudar os processos criativos, onde a causalidade psíquica se sobressai (Figueiredo, 2000).

Depois de Wundt, outras linhas teóricas surgiram e tentaram compreender e explicar o fenômeno psicológico. Porém, de acordo com Bock (2001), todas as abordagens tinham em comum o fato de que procuravam compreender o homem em um aspecto individual: o Comportamentalismo destacando os condicionamentos, a Gestalt investigando as experiências vividas e a Psicanálise enfatizando as forças inconscientes.

Portanto, podemos dizer que a Psicologia enquanto ciência foi caracterizada principalmente por sua capacidade de avaliar os sujeitos. O rompimento com esta dicotomia, de acordo com Bock (2001), surge com a Psicologia SócioHistórica, que, baseada na teoria de Vigotsky, traz uma concepção de homem como ser ativo, social e histórico, que não pode ser separado da realidade social 
e cultural na qual está inserido. Para que se possa compreender o fenômeno psicológico, é necessário entender que "o homem constrói e modifica o mundo e este, por sua vez, propicia os elementos para a constituição psicológica do homem". (Bock, 2001, p. 22).

Considerando esta concepção psicossocial de homem e de mundo, investigar e analisar as características individuais de determinado sujeito sem levar em conta o contexto social e histórico no qual está inserido, é deixar de lado dados fundamentais para que se compreenda a subjetividade do mesmo. É ignorar que seu comportamento, seu pensamento, sua visão de mundo, foram construídos ao longo de sua história, não são inatos, tampouco são estáticos.

Lane (1987) alerta sobre o perigo de pensarmos em uma avaliação que tem como enfoque apenas investigar se determinado sujeito se encaixa dentro de uma classificação nosológica:

"Se assumirmos que somos essencialmente a nossa identidade
social, que ela é a conseqüência de opções que fazemos devido a
nossa constituição bioenergética, ou temperamento, ou mesmo
atrações de personalidade, como aspectos herdados geneticamente,
sem examinarmos as condições sociais que, através da nossa história
pessoal, foram determinando a aquisição dessas características que
nos definem, só poderemos estar reproduzindo o esperado pelos
grupos que nos cercam e julgados 'bem ajustados'" (Lane, 1987, p.
$22-23$ ).

Concordamos com Vigotski (1998), em sua afirmativa que o funcionamento psicológico é fundamentado nas relações sociais entre o indivíduo e o mundo exterior, desenvolvidos em um processo que é social e histórico. Vigotski defendia a ideia de que ao longo do desenvolvimento de suas funções psicológicas superiores, o homem passa a ter a capacidade de expressar e compartilhar seu entendimento individual em relação à experiência comum ao grupo, distinguindo dois componentes do significado da palavra. Um componente seria o significado coletivo, base da compreensão da palavra, compartilhada socialmente. Outro componente seria o sentido da palavra, um significado pessoal, referente ao contexto de uso da palavra e às experiências e vivências afetivas do sujeito em relação a ela (Oliveira, 1997). É este sentido individual que o psicólogo deve tentar captar, utilizando em sua atuação um enfoque que busca apreender o que está implícito na fala dos sujeitos, contextualizando sua realidade.

No entanto, sabemos que ainda há um longo caminho a ser percorrido para que esta concepção se torne uma realidade na práxis psicológica, ou seja, que 
possamos nos aproximar mais desta construção de sentidos, partilhado pelo sujeito. O desafio é pensar em formas de avaliação psicológica que consigam abranger a individualidade sem descaracterizar o contexto social e histórico em sua totalidade. Compreender a subjetividade considerando toda a sua dimensão social e histórica demanda de uma constante reflexão e aperfeiçoamento de técnicas e saberes, pois a realidade é dinâmica, e não podemos esquecer que o próprio observador tem suas próprias concepções, que podem acabar interferindo no fenômeno que é observado.

Dentro da Psicologia existem duas posições em relação à avaliação psicológica: uma critica fortemente os testes psicológicos, concebendo-os como produto de uma visão de homem, sociedade e conhecimento tecnicista, onde os testes psicológicos seriam instrumentos que justificariam os processos de exclusão social:

\begin{abstract}
"Assim, a Psicologia tem contribuído para responsabilizar os sujeitos por seus sucessos e fracassos; [...] temos acreditado que pessoas podem ser classificadas e diferenciadas por suas características e dinâmicas psicológicas; temos criado (ou contribuído para reforçar) padrões de conduta que interessa a sociedade manter, como necessários ao 'bom desenvolvimento das pessoas'. [...] Tem transformado em anormal o diferente, o 'fora do padrão dominante'" (Bock, 2001, p. 25).
\end{abstract}

O grupo divergente entende que a avaliação psicológica baseada em testes representa um conhecimento legítimo, constituindo um critério de avanço do saber embasado cientificamente. (Noronha et al, 2002). Enquadrar os testes psicológicos em uma ótica exclusivamente negativa é ignorar sua importância enquanto instrumento diagnóstico, os estudos realizados e validados no processo de investigação do funcionamento psicológico dos sujeitos.

Sabemos que o comprometimento da atuação do psicólogo deve caminhar na direção da emancipação humana, no respeito à liberdade, à dignidade e na integridade do ser humano. Não é por acaso que um dos princípios fundamentais do atual Código de Ética Profissional do Psicólogo (CFP, 2005) seja basear sua atuação nos valores que embasam a Declaração Universal dos Direitos Humanos. Portanto, podemos pensar que o ideal seria buscar um meio termo entre estes dois posicionamentos, utilizando-se dos instrumentos de avaliação com uma visão que abrange outros elementos constituintes da subjetividade, como os fatores sociais, culturais e ideológicos, possibilitando uma visão global de cada indivíduo. 
A Psicologia Social tem contribuído muito para a transformação da realidade social de nosso país. Nos últimos anos, o profissional de Psicologia tem tido uma participação cada vez maior dentro das políticas públicas, especialmente naquelas voltadas para que a população com menos recursos econômicos tenha acesso a esse cuidado psicossocial, fundamental para o bem-estar. A inclusão do psicólogo nas equipes dos CAPS (Centro de Atendimento Psicossocial), do CRAS (Centro de Referência de Assistência Social), do SUS (Sistema Único de Saúde) e nos programas do SUAS (Sistema Único de Assistência Social) mostram que sua participação é fundamental no processo de inclusão social e de transformação da realidade. Os grupos terapêuticos são um dos fatores fundamentais quando se fala em Saúde Mental e Assistência Social na atualidade. O Ministério do Desenvolvimento Social (2006), em seu guia de orientações técnicas para o Centro de Referência de Assistência Social (CRAS), afirma que o grupo sócioeducativo para as famílias é um excelente espaço para trocas, contribuindo para - exercício da escuta e da fala, da elaboração de dificuldades e de reconhecimento de potencialidades. Contribui para oferecer aos cidadãos a oportunidade de melhor viver os seus direitos dentro de um contexto de proteção mútua, afeto, desenvolvimento pessoal e solidariedade. (Ministério do Desenvolvimento Social, 2006). No entanto, é preciso pensar também neste enfoque dentro da avaliação psicológica, no atendimento individual.

\section{A avaliação psicológica que se quer fazer}

A Psicologia tem avançado de forma significativa na busca por instrumentos e técnicas que consigam compreender os fenômenos psicológicos como sendo resultado de múltiplos fatores. Para chegar a uma conclusão do caso, ou seja, o diagnóstico, o psicólogo analisa e interpreta os dados que obteve ao longo do processo de avaliação psicológica ( informações sobre a queixa inicial, os sintomas apresentados, dados da história clínica, observações do comportamento do paciente durante o processo psicodiagnóstico e os resultados de testes psicométricos e projetivos), de acordo com determinados critérios científicos e diagnósticos. Se certos critérios específicos são atendidos, pode classificar o caso numa categoria nosológica. Para isso, pode-se utilizar de uma das classificações oficiais, como o DSM-IV ou o CID-10. Com base em tal classificação e em aspectos específicos da história clínica, poderá fazer predições sobre o curso 
provável do transtorno (prognóstico) e planejar a intervenção terapêutica adequada. (Cunha, 2000).

A relevância dos aspectos sociais e históricos na construção da história de vida dos sujeitos é tão importante em uma avaliação psicológica que o Conselho Federal de Psicologia criou, em 2003, o Sistema de Avaliação Psicológica (SATEPSI). Este tem como objetivo de qualificar os instrumentos de avaliação, uma vez que muitos deles eram apenas traduções de testes construídos em outros países, que não necessariamente seriam válidos para nossa população (CFP, 2011).

Dentro deste processo de avaliação psicológica ampliada que se propõe, apontamos o uso da entrevista como uma das alternativas eficientes para que se possa ter um acesso diferenciado à realidade do sujeito. É através da fala do indivíduo que poderemos ter acesso à sua subjetividade, ao significado que ele atribui à sua existência, às suas construções subjetivas, experiências e vivências. Com um diálogo onde psicólogo e sujeito tenham a mesma importância, dividindo igualitariamente a responsabilidade na construção de uma compreensão acerca da sua realidade, é que poderemos abrir espaço para um saber compartilhado. É necessário que se tenha o cuidado de conhecer a realidade da população atendida, de procurar entender a sua linguagem e sua cultura e respeitá-las, sem um julgamento prévio do que é certo ou errado ou ainda do que seria melhor para os sujeitos.

"Qualquer trabalho que se proponha a interpretar uma situação altera o campo de forças daquela situação, pois a interpretação é sempre uma escolha em um campo amplo de possibilidades.

$[\ldots]$

O trabalho de avaliação psicológica intervém em vidas singulares.

Como desconsiderar os efeitos que esses trabalhos produzem nos processos de subjetivação se lidamos, exatamente, com esses processos? Eles são da responsabilidade de quem realiza o trabalho de avaliação psicológica"(Machado, 2011).

É importante lembrar que a avaliação psicológica pode ser um importante momento de escuta, uma chance para que o próprio sujeito possa compreender a sua realidade, adquirindo assim uma visão crítica de si mesmo e do mundo, portanto uma ação que pode ser transformadora em si mesma. Talvez uma das possibilidades para uma avaliação psicológica mais próxima da realidade do 
indivíduo atendido seja que os instrumentos de avaliação sejam utilizados pelos psicólogos como um meio de facilitar o diálogo com o paciente.

A partir da coleta de informações, espera-se que cada vez mais os resultados sejam compartilhados com o paciente e que a interpretação destes dados seja construída em uma ação conjunta com o paciente. Desta forma, os significados não serão fornecidos apenas a partir da interpretação estática de um manual de respostas, mas principalmente pela fala e representação atribuídas pelo indivíduo que está sendo avaliado. A proposta que se apresenta nesta perspectiva é de que esta avaliação sirva não apenas para classificar determinado aspecto, mas que possa ser útil para o próprio sujeito, como forma de auto-conhecimento e meio de apropriar-se de sua própria história.

\section{CONCLUSÃO}

O desafio para os psicólogos que atuam e para os que estão em processo de formação é de aprimorar a avaliação psicológica, assim como as demais formas de atuação, visando contribuir cada vez mais para transformar nossa realidade social em um mundo menos desigual. Essa mudança começa na formação acadêmica dos futuros profissionais, com a preocupação em relação à qualificação do uso de testes e técnicas psicológicas, da constante atualização, dos cuidados éticos agregados a discussões, reflexões e a participação ativa dos futuros profissionais, com o objetivo de desenvolver uma visão crítica e atuante, que vai além da teoria e se fundamenta na prática comprometida com a sociedade.

Os profissionais de Psicologia devem estar comprometidos com a garantia dos direitos para os mais diversos segmentos sociais, especialmente aqueles que são excluídos socialmente e não têm acesso aos seus direitos básicos. É necessário que se tenha a consciência de que a cada vez que um direito fundamental é respeitado, toda a sociedade avança na direção do respeito às particularidades de cada indivíduo.

Lane (1987), ao comentar a harmonia encontrada em um hospital psiquiátrico de Cuba, onde todos os envolvidos (pacientes, familiares e profissionais) participavam ativamente da rotina coletiva, reflete que uma participação ativa e igualitária "só é possível em uma sociedade que se propõe a ser toda ela estruturada em relações comunitárias e onde o cidadão respeitável é aquele que participa de grupos que decidem sobre o comum e que trabalha 
produtivamente para o bem de todos, onde o anti-social é aquele cujas ações visam apenas benefícios próprios, considerando-se melhor que os outros". ( $p$. 74) Se cada um fizer o seu melhor, teremos uma sociedade mais justa para todos.

Espera-se que os testes e demais instrumentos técnicos sejam mais uma ferramenta complementar dentro de um processo humanizado, mediado por uma visão abrangente, que considere os aspectos sociais, históricos e individuais de cada caso. Que o foco de toda intervenção psicológica esteja cada vez mais na promoção da qualidade de vida das pessoas e dos grupos sociais, contribuindo para a eliminação de quaisquer formas de discriminação, exploração, violência, crueldade e opressão, como preconiza o Código de Ética do Psicólogo.

Contribuiremos socialmente enquanto psicólogos quando deixarmos em segundo plano uma visão conservadora, tecnicista, que distancia conhecimento teórico e realidade social, para destacarmos cada vez mais, uma visão crítica, participando da construção de uma sociedade igualitária, que usa a diversidade como instrumento de fortalecimento dos grupos sociais. Como Lane (1987) afirma, o viver em grupo permite o confronto e o reconhecimento da diversidade entre as pessoas, aonde cada um vai construindo sua identidade social a partir das interações. Essa atuação comprometida com o respeito e a promoção da liberdade, da dignidade, da igualdade e da integridade do ser humano, como postula o Código de Ética do Psicólogo, independe de qual seja o referencial teórico ou a área de atuação do psicólogo, pois como Eizirik (1988, apud Zanella, 1998, p. 222) afirma:

"Não é o lugar que define a postura de um profissional - embora nem todos pensem assim - é antes a capacidade de refletir criticamente sobre teorias, métodos e práticas, avaliando resultados e pensando acerca das necessidades do país em que nos encontramos".

Ao fazer uma avaliação psicológica ou atender a outra demanda em nossa atuação, talvez seja interessante priorizar o fato de que estamos diante de uma vivência única, não apenas um sujeito de determinado grupo (uma criança com problemas de aprendizagem, um idoso, um paciente da saúde mental, um homossexual), mas um ser humano, com suas experiências, com uma construção de sentidos adquirida em sua trajetória, com suas representações, e que tentar encaixa-lo em determinada classificação é ignorar sua individualidade.

Quando os resultados da avaliação psicológica são compartilhados com a principal pessoa interessada, que é a pessoa avaliada, e a partir desses dados for 
construída com o sujeito uma nova concepção de si mesmo em relação ao mundo, o psicólogo está contribuindo para a sociedade. A avaliação psicológica pode ser a abertura para novas possibilidades, contribuindo para 0 fortalecimento das potencialidades e autonomia dos sujeitos, instrumentalizandoos para o enfrentamento de suas dificuldades e contribuindo de forma significativa para a consolidação de uma sociedade onde todos são respeitados e têm o mesmo direito a uma vida plena de possibilidades.

\section{REFERÊNCIAS}

Bock, A. M. B. (2001). A psicologia sócio-histórica: Uma perspectiva crítica em psicologia. In A. M. B. Bock, M. G. M. Gonçalves, \& O. Furtado (Orgs.) Psicologia sócio-histórica: Uma perspectiva crítica em Psicologia ( $\mathrm{pp}$. 1535). São Paulo: Cortez.

Brasil (2006). NOB-RH\SUAS. Ministério do Desenvolvimento Social e Combate à Fome. Brasília: MDS \SNAS.

Conselho Federal de Psicologia. (2005). Código de Ética Profissional do Psicólogo. Brasília.

Conselho Federal de Psicologia. (2007). Resolução no. 007 de 2003. Brasília.

Disponível em

http://www2.pol.org.br/satepsi/CD testes/pdf/Resolu\%E7\%E30\%20CFP\%2 ON\%BA\%20007-2003.pdf.

Conselho Federal de Psicologia (2011). Ano da avaliação psicológica: Textos geradores. Brasília. Disponível em http://www.pol.org.br/pol/cms/pol/publicacoes/publicacoesDocumentos/ano daavaliacaopsicologica prop8.pdf.

Cunha, J. A. et al (2000). Psicodiagnóstico $V$. 5a. edição revisada e ampliada. Porto Alegre: Artmed.

Figueiredo, L. C. M. (2000). Psicologia, uma (nova) introdução. 2a. Edição. São Paulo: Educ.

Lane, S. T. M. (1987). O que é psicologia social. 13a. Edição. São Paulo: Brasiliense. 
Machado, A. M. (2011). Avaliação psicológica e as relações institucionais. In: Conselho Federal de Psicologia. Ano da avaliação psicológica: Textos geradores. Brasília: Conselho Federal de Psicologia. Disponível em http://www.pol.org.br/pol/cms/pol/publicacoes/publicacoesDocumentos/ano daavaliacaopsicologica prop8.pdf.

Noronha, Ana Paula et al (2002). Em defesa da avaliação psicológica. Avaliação psicológica, 1(2), 173-174.

Oliveira, M. K. (1997). Vygotsky - aprendizado e desenvolvimento: Um processo sócio-histórico. São Paulo: Scipione.

Vigotsky, L. S. (1998). A formação social da mente: O desenvolvimento dos processos psicológicos superiores. 6a. Edição. São Paulo: Martins Fontes.

Zanella, A. V. (1998). Psicologia Social e Escola. In: Strey, M. N. et al. Psicologia Social contemporânea. (p. 221-229). Petrópolis, RJ: Vozes.

Contato: lucianacescon@yahoo.com.br

Recebido em: 15/05/2013

Revisado em: 20/06/2013

Aceito em: 13/09/2013 